\title{
A NOTE ON FIBONACCI MATRICES OF EVEN DEGREE
}

\author{
MICHELE ELIA
}

(Received 1 March 2001)

\begin{abstract}
This paper presents a construction of $m$-by- $m$ irreducible Fibonacci matrices for any even $m$. The proposed technique relies on matrix representations of algebraic number fields which are an extension of the golden section field. The explicit construction of some 6-by-6 and 8-by-8 irreducible Fibonacci matrices is given.
\end{abstract}

2000 Mathematics Subject Classification. 11B39, 15A36.

1. Introduction. Fibonacci matrices are characterized by the property that all entries in their positive powers are positive or negative Fibonacci numbers. In general, products of Fibonacci matrices that are not powers of a single matrix, are not Fibonacci matrices. Nevertheless, in [1], Dazheng introduced a set of 4-by-4 Fibonacci matrices which was closed for matrix multiplication. In the same paper, the search for existence conditions and construction of $m$-by- $m$ Fibonacci matrices was proposed as an open problem for every $m>4$. A special class of 4-by-4 Fibonacci matrices had previously been considered in [3].

In [9], Washington showed that all 4-by-4 Fibonacci matrices described in [1] belong to a matrix representation of the group of units in $\mathbb{Z}\left[\zeta_{5}\right]$, the integral ring of the cyclotomic field $\mathbb{Q}\left(\zeta_{5}\right)$ (see [8]). Incidentally, the golden section ring $\mathbb{Z}[\omega]$ with $\omega=$ $(1+\sqrt{5}) / 2$ (see [2]), is a subring of $\mathbb{Z}\left[\zeta_{5}\right]$.

Related to the construction of Fibonacci matrices is their classification, namely the enumeration of all finite or infinite classes consisting of the powers of a single matrix which is not a power of another Fibonacci matrix. For the 2-by-2 matrices the classification problem was already solved in [1], and definitively settled in [9]. In the same paper [9], an enumeration of the 4-by-4 Fibonacci matrices was attempted. However, from these results, it turns out that for a Fibonacci matrix classification, the definition of the equivalence notion should include the concepts of rationally irreducible and of periodic matrices.

In this paper, we describe a construction of $m$-by- $m$ Fibonacci matrices for any even $m$. The construction exploits Washington's method in the realm of matrix representations of algebraic number fields, with a key role played by the golden section ring $\mathbb{Z}[\omega]$.

2. General results. The definition of Fibonacci matrices given in [1] is recast to include periodic matrices and an equivalence notion based on matrices over $\mathbb{Q}$. 
DEFINITION 2.1. An $r \times r$ matrix $A$ with integer elements is called a Fibonacci matrix (FM for short) if $A^{n}, n=1,2, \ldots$, has only Fibonacci numbers as its elements, which may be positive, negative, or zero. A Fibonacci matrix $A$ is said to be simple if it is nonsingular, and

(a) no permutation matrix $P$ and integer $m$ exist such that $P A^{m} P^{-1}$ is a block diagonal matrix of Fibonacci submatrices,

(b) $\left\{A^{n} \mid n \geq 0\right\}$ has entries $F_{i} \in A^{n}$ for all $i$ and some $n$.

A simple Fibonacci matrix $A$ is said to be an irreducible FM if no rational matrix $U$ exists such that $U A U^{-1}$ is a block diagonal matrix of Fibonacci submatrices.

A Fibonacci matrix $A$ is called basic if its elements are only $1,-1$, and 0 .

This definition associates to any Fibonacci matrix $A$ a sequence of matrices $I, A$, $A^{2}, \ldots, A^{n}, \ldots$, and subordinately $r^{2}$ scalar sequences that contain only Fibonacci numbers. The matrix sequence satisfies the first order linear matrix recurrence

$$
X(n+1)=A X(n)
$$

with initial condition $X(0)=I$. This equation is equivalent to a linear recurrence

$$
x(n+r)=\sum_{i=0}^{r-1} a_{r-i} x(n+i)
$$

of order $r$, whose characteristic polynomial $p(\lambda)$ is the characteristic polynomial $\operatorname{det}(\lambda I-A)$ of matrix $A$. Using the distinct roots $\lambda_{1}, \lambda_{2}, \ldots, \lambda_{u}$ of $p(\lambda)$, we can write

$$
x(n)=\sum_{i=1}^{u} c_{i}(n) \lambda_{i}^{n}
$$

where $c_{i}(n)$ is a polynomial in $n$ of degree one less than the multiplicity of $\lambda_{i}$. If $c_{i}(n) \neq 0$ then $\lambda_{i}$ is said to be active, otherwise it is said to be inactive.

Let $\mathbb{Z}_{t}^{*}$ denote the multiplicative group of residues modulo $t$. The order of $\mathbb{Z}_{t}^{*}$ is $\varphi(t)$, the Euler totient function. A linear recurrence producing an infinite sequence that contains only Fibonacci numbers is characterized by the following theorem.

THEOREM 2.2. Let $\mathfrak{s}$ be an infinite sequence $x(0), x(1), \ldots, x(n), \ldots$ generated by a linear recurrence (2.2) of order $r(\geq 2)$ with integer coefficients and having characteristic polynomial $p(\lambda)$. If $\mathfrak{s}$ is an unbounded sequence containing only Fibonacci numbers, then

(1) the active root of $p(\lambda)$ of largest absolute value is $\omega^{s} \zeta_{t}$ for some integer $s$ and $\zeta_{t}$ denotes a th primitive root of unity;

(2) $p(\lambda)$ is a polynomial over $\mathbb{Q}$ which is divisible by the polynomial

$$
m(\lambda)=\prod_{i \in \mathbb{Z}_{t}^{*}}\left(\lambda-\omega^{s} \zeta_{t}^{i}\right)\left(\lambda-\bar{\omega}^{s} \zeta_{t}^{i}\right)=m_{t}\left(\frac{\lambda}{\omega^{s}}\right) m_{t}\left(\frac{\lambda}{\bar{\omega}^{s}}\right),
$$

where $m_{t}(\lambda)$ is the th cyclotomic polynomial of degree $\varphi(t)$ and $m(\lambda)$ is irreducible over $\mathbb{Q}$. 
Proof. Since $\mathfrak{s}$ is unbounded and $x(n)$ has the form (2.3), the largest absolute value $\left|\lambda_{\max }\right|$ of the roots of $p(\lambda)$ is greater than 1 . Then, a subsequence $\tilde{\mathfrak{s}}$ of $\mathfrak{s}$ exists (see [5, Theorem 3.6, page 45]), such that

$$
\lim _{n \rightarrow \infty}\left|\frac{\tilde{x}(n+1)}{\tilde{x}(n)}\right|=\left|\lambda_{\max }\right|=\omega^{s}
$$

for some integer $s$. Thus, $\lambda_{\max }=\omega^{s} \zeta_{t}$, where $\zeta_{t}$ is a primitive $t$ th root of unity. In conclusion, the polynomial $m(\lambda)$ defined in (2.4) with roots $\omega^{s} \zeta_{t}^{i}, i \in \mathbb{Z}_{t}^{*}$, has integer coefficients, is irreducible over $\mathbb{Q}$ and has a root in common with $p(\lambda)$. By a Lagrange theorem, $m(\lambda)$ divides $p(\lambda)$.

THEOREM 2.3. Let $A$ be an $r \times r$ nonsingular Fibonacci matrix, therefore

(i) If the entries in each matrix $A^{n}, n=1,2, \ldots$, are bounded, then $A$ generates a cyclic group of finite order (this property was already observed by Kronecker [4, page 24 ] in another context) $N$, with $\varphi(N)=r / \ell$ for some divisor $\ell$ of $r$.

(ii) If $A$ is a simple $F M$, then its characteristic polynomial $p(\lambda)$ is divisible by $m(\lambda)=$ $m_{t}\left(\lambda / \omega^{s}\right) m_{t}\left(\lambda / \bar{\omega}^{s}\right)$ for suitable $s$ and $t$.

Proof. If the entries in each matrix $A^{n}$ are bounded, then only a finite number of distinct matrices exists. Thus, there is a minimum integer $N$ such that $A^{N}=I_{r}$. Therefore, $A$ generates a cyclic group of order $N$. Let $\phi(\lambda)$ be the minimal polynomial of $A$, therefore $\phi(\lambda)$ is a factor of both $\lambda^{N}-1$ and the characteristic polynomial $p(\lambda)$ of $A$. It follows that $N$ satisfies the condition $r=\ell \varphi(N)$, and is maximum when $\varphi(N)=r$.

If $A$ is a simple FM then some of its entries belong to a recurrent sequence of unbounded Fibonacci numbers; the conclusion follows from Theorem 2.2, since the characteristic polynomial of these sequences is $p(\lambda)$.

2.1. Fibonacci matrices of even degree. In view of Theorem 2.3, the splitting field of the characteristic polynomial of any aperiodic FM matrix contains the subfield $\mathbb{Q}(\omega)$. In particular, the characteristic polynomial of irreducible FM is specified by the following theorem.

THEOREM 2.4. Any $2 r \times 2 r \mathbb{Q}$-irreducible Fibonacci matrix $M$ can be obtained from the natural representation of a ring $\mathbb{Z}[\eta]$ having $\mathbb{Z}[\omega]$ as a subring. $\eta$ is a root of an irreducible polynomial $m(\lambda)$ of degree $2 r$, which splits over $\mathbb{Z}[\omega]$ into two irreducible factors of degree $r$.

PRoof. Irreducible matrices have irreducible characteristic polynomials. Therefore, the characteristic polynomial $m(\lambda)$ of $M$ with integer coefficients is irreducible over $\mathbb{Q}$. By Theorem 2.3, it is divided by the irreducible polynomial $g(\lambda, \omega) g(\lambda, 1-\omega)$ with roots $\zeta_{t}^{i} \omega^{s}$, for some integers $s$ and $t$. It follows that $m(\lambda)=g(\lambda, \omega) g(\lambda, 1-\omega)$, moreover, $M$ is rationally equivalent to a matrix $\rho\left(\zeta_{t} \omega^{s}\right)$, in other words there is a basis in $\mathbb{Z}[\eta]$ such that $M$ is a representation of $\zeta_{t} \omega^{s}$. 
THEOREM 2.5. Let $\rho$ be the natural representation of $\mathbb{Z}[\eta]$, where $\eta$ is a root of $m(\lambda)$, then $\rho(\omega)$ is a matrix that satisfies the minimal equation $\rho(\omega)^{2}-\rho(\omega)-I=O$. The characteristic polynomial of $\rho(\omega)$ is $\left(\lambda^{2}-\lambda-1\right)^{r}$, and $\rho(\omega)$ can be diagonalized in $\mathbb{Z}[\eta]$. The powers $\rho(\omega)^{n}$ can be computed as

$$
\rho(\omega)^{n}=F_{n-1} I_{r}+F_{n} \rho(\omega) .
$$

If $\rho(\omega)$ is basic, then it is an FM.

Proof. The assertion about the minimal equation for $\rho(\omega)$ is proved by applying $\rho$ to the equation $\omega^{2}-\omega-1=0$. Since $\rho(\eta)$ can be diagonalized in $\mathbb{Z}[\eta]$, the same holds for $\rho(\omega)$, which is a linear combination of powers of $\rho(\eta)$.

The characteristic roots of $\rho(\omega)$ must be either $\omega$ or its conjugate, therefore the conclusion follows because the coefficients of the characteristic polynomial belong to $\mathbb{Q}$.

Since it is well known that $\omega^{n}=F_{n-1}+F_{n} \omega$, the assertion is proved by the application of $\rho$.

In general, if $\rho(\omega)$ is not basic, it may or may not be an FM as shown by the following counterexample. It is well known that the matrix

$$
M_{1}=\left[\begin{array}{ll}
0 & 1 \\
1 & 1
\end{array}\right]
$$

is an FM. The following equivalent matrix

$$
\left[\begin{array}{ll}
-1 & 1 \\
-1 & 2
\end{array}\right]=\left[\begin{array}{ll}
1 & 0 \\
1 & 1
\end{array}\right]\left[\begin{array}{ll}
0 & 1 \\
1 & 1
\end{array}\right]\left[\begin{array}{cc}
1 & 0 \\
-1 & 1
\end{array}\right],
$$

although not basic, is an FM, whereas the following equivalent matrix

$$
\left[\begin{array}{ll}
-2 & 1 \\
-5 & 3
\end{array}\right]=\left[\begin{array}{ll}
1 & 0 \\
2 & 1
\end{array}\right]\left[\begin{array}{ll}
0 & 1 \\
1 & 1
\end{array}\right]\left[\begin{array}{cc}
1 & 0 \\
-2 & 1
\end{array}\right]
$$

is not an FM because its square

$$
\left[\begin{array}{ll}
-2 & 1 \\
-5 & 3
\end{array}\right]^{2}=\left[\begin{array}{ll}
-1 & 1 \\
-5 & 4
\end{array}\right]
$$

has an entry that is not a Fibonacci number.

Washington [9] has shown that all Fibonacci matrices introduced by Dazheng [1] belong to an integral representation of the ring $\mathbb{Z}\left[\zeta_{5}\right]$. However, this property is not peculiar of all Fibonacci matrix as shown for example by the tridiagonal matrix found in [3]. In particular, $A_{F}$ and the matrix $\bar{F}_{10}$ defined in [1] are equivalent in $\mathbb{Q}$ to different 
block diagonal FMs, that is,

$$
\begin{aligned}
& {\left[\begin{array}{cccc}
1 & 1 & 1 & 1 \\
1 & 2 & 2 & 1 \\
1 & 1 & -1 & -1 \\
-1 & 0 & 0 & 1
\end{array}\right]\left[\begin{array}{llll}
0 & 1 & 0 & 0 \\
1 & 0 & 1 & 0 \\
0 & 1 & 0 & 1 \\
0 & 0 & 1 & 0
\end{array}\right]\left[\begin{array}{cccc}
1 & -\frac{1}{2} & 0 & -\frac{1}{2} \\
-\frac{1}{2} & \frac{1}{2} & \frac{1}{2} & \frac{1}{2} \\
-\frac{1}{2} & \frac{1}{2} & -\frac{1}{2} & -\frac{1}{2} \\
1 & -\frac{1}{2} & 0 & \frac{1}{2}
\end{array}\right]} \\
& =\left[\begin{array}{cccc}
0 & 1 & 0 & 0 \\
1 & 1 & 0 & 0 \\
0 & 0 & 0 & -1 \\
0 & 0 & -1 & -1
\end{array}\right], \\
& {\left[\begin{array}{cccc}
1 & 0 & 0 & 0 \\
0 & 0 & 1 & 1 \\
1 & 3 & 0 & 1 \\
-2 & -2 & -2 & 1
\end{array}\right]\left[\begin{array}{cccc}
0 & 0 & -1 & -1 \\
1 & 1 & 1 & 0 \\
0 & 1 & 1 & 1 \\
-1 & -1 & 0 & 0
\end{array}\right]\left[\begin{array}{cccc}
1 & 0 & 0 & 0 \\
-\frac{5}{11} & -\frac{2}{11} & \frac{3}{11} & -\frac{1}{11} \\
-\frac{4}{11} & \frac{5}{11} & -\frac{2}{11} & -\frac{3}{11} \\
\frac{4}{11} & \frac{6}{11} & \frac{2}{11} & \frac{3}{11}
\end{array}\right]} \\
& =\left[\begin{array}{cccc}
0 & -1 & 0 & 0 \\
-1 & 1 & 0 & 0 \\
0 & 0 & 0 & -1 \\
0 & 0 & -1 & 1
\end{array}\right] .
\end{aligned}
$$

The matrix $A_{F}$ does not belong to a representation. In fact, it has four distinct eigenvalues $\omega, 1-\omega,-\omega$, and $-1+\omega$, and its minimal polynomial has degree 4 . This is in contrast to Theorem 2.5, which implies that any reducible FM belonging to a ring representation has minimal polynomial $\lambda^{2}-\lambda-1$.

THEOREM 2.6. A matrix $\rho(\zeta \omega)$, belonging to a representation of an algebraic number field $\mathbb{Q}(\eta)$, is a Fibonacci matrix only if $\zeta \in \mathbb{Z}[\eta]$ is a root of unity. If $\eta=\zeta_{m}$, then $\mathbb{Q}(\eta)$ is a cyclotomic field, and necessarily $5 \mid m$.

Proof. The eigenvalues of $\rho(\zeta \omega)$ are $\zeta \omega$ and its conjugates, which are the roots of the characteristic equation of any recurrence satisfied by the entries of this matrix. Therefore, Theorem 2.2 implies the existence of an integer $s(\geq 2)$ such that $(\zeta \omega)^{s}=$ $\omega^{s}$. It follows that $\zeta^{s}=1$ and $\zeta$ is a root of unity, which belongs to the torsion part of the group of units of $\mathbb{Z}[\eta]$.

If $\eta=\zeta_{m}$, then $\mathbb{Q}(\eta)$ is a cyclotomic field. Since $\omega \in \mathbb{Q}\left(\zeta_{m}\right)$, then $\mathbb{Q}(\omega) \subset \mathbb{Q}\left(\zeta_{m}\right)$. It is known that $\mathbb{Q}(\omega) \subseteq \mathbb{Q}\left(\zeta_{5}\right)$, thus $\mathbb{Q}(\omega) \subset \mathbb{Q}\left(\zeta_{m}\right) \cap \mathbb{Q}\left(\zeta_{5}\right)$. The conclusion 5 । $m$ follows from [8, Proposition 2.4, page 11], which states that if $(m, n)=1$ then $\mathbb{Q}\left(\zeta_{m}\right) \cap \mathbb{Q}\left(\zeta_{n}\right)=\mathbb{Q}$. 
2.2. Constructions. Theorem 2.4 has also constructive aspects and is useful in the classification of Fibonacci matrices. Although not elegant, the following construction shows the existence of FM for any $2 n$. Consider the following recursive procedure on matrix dimension which is started with matrix $M_{1}$. Let $A \oplus B$ denote a diagonal block matrix with blocks $A$ and $B$. Let $T_{n}$ be a $2 n \times 2 n$ matrix with all $1 s$ in the main diagonal, $a_{n 1}=1$ and all other entries equal 0 . The sequence $M_{1}, M_{2}, \ldots$ of FMs of increasing degree is defined as

$$
M_{n}=T_{n}\left(M_{n-1} \oplus M_{1}\right) T_{n}^{-1}
$$

It is immediately seen that $M_{n}$ is basic, and satisfies the minimal equation $M_{n}^{2}-M_{n}-$ $I=O$, therefore $M_{n}$ is a simple FM. In fact, it is not in block-diagonal form and cannot be transformed into such a form by a permutation matrix.

The construction of irreducible FM is a more difficult task, and Theorem 2.4 is again useful. Let $m_{1}(\lambda, \omega)$ be an irreducible polynomial of degree $r$ over $\mathbb{Z}[\omega]$ with some coefficient effectively in $\mathbb{Z}[\omega]$. For example, the polynomials $\lambda^{r}-\omega$ and $\lambda^{2 r}-\omega \lambda^{r}+$ 1 are irreducible in $\mathbb{Z}[\omega]$ for any $r$, while $\lambda^{r}-\omega \lambda+1$ is irreducible for any $r$ up to 11 . The polynomials $m(\lambda)=m_{1}(\lambda, \omega) m_{1}(\lambda, 1-\omega)$ are irreducible of degree $2 r$ with coefficients in $\mathbb{Z}$. Therefore, the representations of the related algebraic number fields possibly contain FMs. The method is illustrated by the following two constructions of irreducible FMs of degrees 6 and 8.

2.3. $6 \times 6$ matrices. An irreducible $6 \times 6$ matrix can be constructed from an algebraic field of degree 6 having subfield $\mathbb{Q}(\omega)$. Let $\eta$ be a root of the irreducible polynomial over $\mathbb{Q}$

$$
m_{i 6}(x)=x^{6}+x^{4}+2 x^{3}-x^{2}+x+1=\left(x^{3}+\omega x+1\right)\left(x^{3}+(1-\omega) x+1\right)
$$

which generates the field $\mathbb{Q}(\eta)$. The matrix is built with the same rule that is given by Washington [9]

$$
\begin{aligned}
\omega & =1-\eta+\eta^{2}+\eta^{3}+\eta^{5} ; \quad \omega \eta^{3}=-\eta^{2}-\eta^{5} ; \\
\omega \eta & =-1-\eta^{3} ; \quad \omega \eta^{4}=1+\eta-\eta^{2}+\eta^{3}+\eta^{4} ; \\
\omega \eta^{2} & =-\eta-\eta^{4} ; \quad \omega \eta^{5}=\eta+\eta^{2}-\eta^{3}+\eta^{4}+\eta^{5} ;
\end{aligned}
$$

and results

$$
\rho(\omega)=\left[\begin{array}{cccccc}
1 & -1 & 1 & 1 & 0 & 1 \\
-1 & 0 & 0 & -1 & 0 & 0 \\
0 & -1 & 0 & 0 & -1 & 0 \\
0 & 0 & -1 & 0 & 0 & -1 \\
1 & 1 & -1 & 1 & 1 & 0 \\
0 & 1 & 1 & -1 & 1 & 1
\end{array}\right]
$$

2.4. $8 \times 8$ matrices. An $8 \times 8$ matrix can be constructed from the cyclotomic field $\mathbb{Q}\left[\zeta_{15}\right]$ of degree 15 which has the subfield $\mathbb{Q}(\omega)$. This example has the same 
characteristic as the FM of degree 4 constructed by Dazheng [1]. The cyclotomic polynomial $m_{15}(x)$ has degree 8 and factors over $\mathbb{Q}(\omega)$ into two irreducible polynomials of degree 4

$$
\begin{aligned}
m_{15}(x)= & 1-x+x^{3}-x^{4}+x^{5}-x^{7}+x^{8} \\
= & \left(x^{4}-\omega x^{3}+\omega x^{2}-\omega x+1\right) \\
& \times\left(x^{4}-(1-\omega) x^{3}+(1-\omega) x^{2}-(1-\omega) x+1\right) .
\end{aligned}
$$

In terms of $\zeta_{15}$ we have $\omega=1-\zeta_{15}^{2}+\zeta_{15}^{3}-\zeta_{15}^{7}$, therefore we obtain the Fibonacci matrix

$$
\begin{aligned}
\rho(\omega) & =\left[\begin{array}{cccccccc}
1 & 0 & -1 & 1 & 0 & 0 & 0 & -1 \\
1 & 0 & 0 & 0 & 0 & 1 & 0 & -1 \\
1 & 0 & 0 & 1 & -1 & 1 & 1 & -1 \\
1 & 0 & 0 & 1 & 0 & 0 & 1 & 0 \\
0 & 1 & 0 & 0 & 1 & 0 & 0 & 1 \\
-1 & 1 & 1 & -1 & 1 & 0 & 0 & 1 \\
-1 & 0 & 1 & 0 & 0 & 0 & 0 & 1 \\
-1 & 0 & 0 & 0 & 1 & -1 & 0 & 1
\end{array}\right], \\
\rho\left(\zeta_{15}\right) & =\left[\begin{array}{cccccccc}
0 & 1 & 0 & 0 & 0 & 0 & 0 & 0 \\
0 & 0 & 1 & 0 & 0 & 0 & 0 & 0 \\
0 & 0 & 0 & 1 & 0 & 0 & 0 & 0 \\
0 & 0 & 0 & 0 & 1 & 0 & 0 & 0 \\
0 & 0 & 0 & 0 & 0 & 1 & 0 & 0 \\
0 & 0 & 0 & 0 & 0 & 0 & 1 & 0 \\
0 & 0 & 0 & 0 & 0 & 0 & 0 & 1 \\
-1 & 1 & 0 & -1 & 1 & -1 & 0 & 1
\end{array}\right] .
\end{aligned}
$$

In this case we obtain 15 Fibonacci matrices as $\rho\left(\zeta_{15}^{j} \omega\right), j=0,1, \ldots, 15$. For instance, we have

$$
\rho\left(\zeta_{15} \omega\right)=\left[\begin{array}{cccccccc}
1 & 0 & 0 & 0 & 0 & 1 & 0 & -1 \\
1 & 0 & 0 & 1 & -1 & 1 & 1 & -1 \\
1 & 0 & 0 & 1 & 0 & 0 & 1 & 0 \\
0 & 1 & 0 & 0 & 1 & 0 & 0 & 1 \\
-1 & 1 & 1 & -1 & 1 & 0 & 0 & 1 \\
-1 & 0 & 1 & 0 & 0 & 0 & 0 & 1 \\
-1 & 0 & 0 & 0 & 1 & -1 & 0 & 1 \\
-1 & 0 & 0 & -1 & 1 & 0 & -1 & 1
\end{array}\right]
$$

Note that the group of the units in $\mathbb{Z}\left(\zeta_{15}\right)$ is isomorphic to $\mathbb{Z}^{3} \times C_{15}$ by Dirichlet's theorem [6], therefore it is generated by $\rho(\omega), \rho\left(-\zeta_{15}\right)$, and by the two following matrices: $\rho\left(\zeta_{15}^{7}+\zeta_{15}^{-7}\right)$ and $\rho\left(\zeta_{15}^{6}+\zeta_{15}^{-6}+\zeta_{15}^{7}+\zeta_{15}^{-7}\right)$ which cannot be FM. In this case the FM matrices form only a subgroup of the units in $\mathbb{Z}\left(\zeta_{15}\right)$. 
TABLE 3.1. Solutions of $\phi(N)=k$ with $k \leq 4$, (see [7, page 250]).

\begin{tabular}{ll}
\hline$k$ & $N: \phi(N)=k$ \\
\hline 1 & 1,2 \\
2 & $3,4,6$ \\
3 & - \\
4 & $5,8,10,12$ \\
\hline
\end{tabular}

3. FM classification. In the following, we complete the classification of Fibonacci matrices of degree 2 given in [9] including periodic matrices, and outline the classification of 4 degree matrices.

Periodic and aperiodic FM will be considered separately. The periodic matrices have period $N$ if and only if all eigenvalues are $N$ th roots of unity. Number fields related to the construction of aperiodic FMs always contain $\mathbb{Q}(\omega)$ by Theorem 2.6, a condition which is not indispensable for number fields related to periodic Fibonacci matrices.

\subsection{Periodic matrices}

3.1.1. $2 \times 2$ FM. The characteristic polynomial $p(\lambda)$ of a $2 \times 2$ periodic matrix must be the product of cyclotomic polynomials of degree $\phi(N)$ not greater than 2 . The solutions of $\phi(N)=k$ with $k \leq 2$, taken from Table 3.1, indicate that possible periods are $6,4,3$, and 2 .

We have infinite classes of matrices of period 2 and period 4

$$
M_{2}=\left[\begin{array}{cc}
F_{2 n+1} & -F_{2 n} \\
F_{2 n+2} & -F_{2 n+1}
\end{array}\right], \quad M_{4}=\left[\begin{array}{cc}
F_{2 n} & -F_{2 n-1} \\
F_{2 n+1} & F_{2 n}
\end{array}\right] \text {. }
$$

Matrices of period 3 and 6 are of the form

$$
M_{3}=\left[\begin{array}{cc}
a & b \\
-\frac{1 \pm a-a^{2}}{b} & \pm 1-a
\end{array}\right]
$$

where plus and minus signs yield matrices of period 6 and 3, respectively. Therefore, 12 FMs of period 6 are obtained for $(a, b) \in\{(-1, \pm 1),(-1, \pm 3),(0, \pm 1),(1, \pm 1),(2, \pm 1)$, $(2, \pm 3)\}$; and 12 FMs of period 3 are obtained for $(a, b) \in\{(-2, \pm 1),(-2, \pm 3),(-1, \pm 1)$, $(0, \pm 1),(1, \pm 1),(1, \pm 3)\}$.

3.1.2. $4 \times 4$ FM. The characteristic polynomial $p(\lambda)$ of a $4 \times 4$ periodic matrix must be the product of cyclotomic polynomials of degree $\phi(N)$ not greater than 4 . In particular, from Table 3.1 it is deduced that possible periods are 2, 3, 4, 5, 6, 8, 10, and 12 .

PERIOD 2. Since there are four distinct classes of nonequivalent diagonal matrices of order 2 , namely $\operatorname{diag}(1,1,1,-1), \operatorname{diag}(1,1,-1,-1), \operatorname{diag}(1,-1,-1,-1)$, and $\operatorname{diag}(-1,-1,-1,-1)$, there will be four classes of FM of period 2 . We can have an infinite number of FM of period 2, whereas for larger periods only a finite number of FMs is possible. In the following only one example for each period is given. 
PERIODS 3 AND 4. The matrix $\rho\left(\zeta_{3}\right)$ with characteristic polynomial $\left(x^{2}+x+1\right)^{2}$ has period 3 , and represents the cube root of unity in the ring of integers $\mathbb{Z}[\alpha]$, with $\alpha$ a root of $x^{4}-2 x^{3}+2 x^{2}-x+1$. The matrix $\rho\left(\zeta_{4}\right)$ with characteristic polynomial $x^{4}-1$ has period 4 ,

$$
\rho\left(\zeta_{3}\right)=\left[\begin{array}{cccc}
0 & -1 & 1 & 0 \\
0 & 0 & -1 & 1 \\
-1 & 1 & -2 & 1 \\
-1 & 0 & -1 & 0
\end{array}\right], \quad \rho\left(\zeta_{4}\right)=\left[\begin{array}{llll}
0 & 1 & 0 & 0 \\
0 & 0 & 1 & 0 \\
0 & 0 & 0 & 1 \\
1 & 0 & 0 & 0
\end{array}\right] .
$$

PERIODS 5 AND 6. The matrix $\rho\left(\zeta_{5}\right)$ with characteristic polynomial $x^{4}+x^{3}+x^{2}+$ $x+1$ has period 5 . The matrix $\rho\left(\zeta_{6}\right)$ with characteristic polynomial $\left(x^{2}-x+1\right)^{2}$ has period 6 ,

$$
\rho\left(\zeta_{5}\right)=\left[\begin{array}{cccc}
0 & 1 & 0 & 0 \\
0 & 0 & 1 & 0 \\
0 & 0 & 0 & 1 \\
-1 & -1 & -1 & -1
\end{array}\right], \quad \rho\left(\zeta_{6}\right)=\left[\begin{array}{cccc}
0 & 1 & -1 & 0 \\
0 & 0 & 1 & -1 \\
1 & -1 & 2 & -1 \\
1 & 0 & 1 & 0
\end{array}\right]
$$

PERIODS 8 AND 10. The matrix $\rho\left(\zeta_{8}\right)$ with characteristic polynomial $x^{4}+1$ has period 8 . The matrix $\rho\left(\zeta_{10}\right)$ with characteristic polynomial $x^{4}-x^{3}+x^{2}-x+1$ has period 10,

$$
\rho\left(\zeta_{8}\right)=\left[\begin{array}{cccc}
0 & 1 & 0 & 0 \\
0 & 0 & 1 & 0 \\
0 & 0 & 0 & 1 \\
-1 & 0 & 0 & 0
\end{array}\right], \quad \rho\left(\zeta_{10}\right)=\left[\begin{array}{cccc}
0 & 1 & 0 & 0 \\
0 & 0 & 1 & 0 \\
0 & 0 & 0 & 1 \\
-1 & 1 & -1 & 1
\end{array}\right] .
$$

PERIOD 12. The matrices

$$
\left[\begin{array}{cccc}
0 & 1 & 0 & 0 \\
0 & 0 & 1 & 0 \\
0 & 0 & 0 & 1 \\
-1 & 0 & 1 & 0
\end{array}\right], \quad\left[\begin{array}{cccc}
0 & 1 & 0 & 0 \\
0 & 0 & 1 & 0 \\
0 & 0 & 0 & 1 \\
-1 & -1 & -2 & -1
\end{array}\right]
$$

have period 12 and characteristic polynomials $x^{4}-x^{2}+1$ and $\left(x^{2}+1\right)\left(x^{2}+x+1\right)=$ $x^{4}+x^{3}+2 x^{2}+x+1$, respectively.

3.2. Aperiodic matrices. In [9], Washington proved that there are only 8 classes of $2 \times 2$ FM equivalent to each of the two classes of nonequivalent FM generated by

$$
\left[\begin{array}{ll}
0 & 1 \\
1 & 1
\end{array}\right], \quad\left[\begin{array}{cc}
0 & -1 \\
-1 & -1
\end{array}\right],
$$

respectively. For $4 \times 4$ aperiodic Fibonacci matrices the situation is more complex, and it is necessary to distinguish irreducible and reducible matrices. 
The characteristic polynomials of irreducible Fibonacci matrices can be

$$
\begin{array}{ll}
t=3 & x^{4}+L_{s} x^{3}+\left(L_{2 s}+(-1)^{s}\right) x^{2}+(-1)^{s} L_{s} x+1, \\
t=4 & x^{4}+L_{2 s} x^{2}+1, \\
t=5 & x^{4}+L_{s+1} x^{3}+\left(L_{2 s}+(-1)^{s+1}\right) x^{2}+(-1)^{s+1} L_{s-1} x+1,
\end{array}
$$

because a root must be of the form $\omega^{s} \zeta_{t}$, and its conjugates, therefore $t \leq 5$. The case $t=2$ yields reducible polynomials $x^{4}-L_{2 s} x^{2}+1$ to which correspond reducible Fibonacci matrices.

The characteristic polynomials of reducible nonblock diagonal Fibonacci matrices are

$$
\begin{array}{ll}
1 & x^{4}-L_{2 s} x^{2}+1, \\
2 & \left(x^{2} \pm L_{s} x+(-1)^{s}\right)^{2}, \\
3 & \left(x^{2} \pm L_{s} x+(-1)^{s}\right)\left(x^{2} \pm 1\right), \\
4 & \left(x^{2} \pm L_{s} x+(-1)^{s}\right)\left(x^{2} \pm x+1\right) .
\end{array}
$$

In the following we will show one Fibonacci matrix for each polynomial. The number of distinct classes of Fibonacci matrices for each characteristic polynomial may be very large and the problem of computing such a number remains open.

IRREDUCIBLE APERIODIC FM. All irreducible Fibonacci matrices are obtained from a field representation for $t=3,4,5$, and provide a representation of the group of field units with basis $\left\{1, \omega, \zeta_{t}, \omega \zeta_{t}\right\}$. If $t=5$ then a second representation is possible, as Washington showed with basis $\left\{1, \zeta_{5}, \zeta_{5}^{2}, \zeta_{5}^{3}\right\}$.

Let $t=3$, set $s=1$ then the polynomial $x^{4}+x^{3}+2 x^{2}-x+1$ has roots $\omega \zeta_{3}, \bar{\omega} \zeta_{3}$, $\omega \zeta_{3}^{2}$, and $\bar{\omega} \zeta_{3}^{2}$. If, in the field $\mathbb{Z}\left[\omega \zeta_{3}\right]$, we consider the basis $\left\{1, \omega, \zeta_{3}, \omega \zeta_{3}\right\}$, then we have

$$
\rho\left(\omega \zeta_{3}\right)=\rho(\omega) \rho\left(\zeta_{3}\right)=\left[\begin{array}{cccc}
0 & 0 & 0 & 1 \\
0 & 0 & 1 & 1 \\
0 & -1 & 0 & -1 \\
-1 & -1 & -1 & -1
\end{array}\right]
$$

From $\rho\left(\omega \zeta_{3}\right)^{3 m+\epsilon}=\rho(\omega)^{3 m+\epsilon} \rho\left(\zeta_{3}\right)^{\epsilon}$, with $\epsilon=0,1,2$, it follows that $\rho\left(\omega \zeta_{3}\right)$ is an FM since $\rho(\omega)$ is a block diagonal FM and both

$$
\rho\left(\zeta_{3}\right)=\left[\begin{array}{cccc}
0 & 0 & 1 & 0 \\
0 & 0 & 0 & 1 \\
-1 & 0 & -1 & 0 \\
0 & -1 & 0 & -1
\end{array}\right], \quad \rho\left(\zeta_{3}^{2}\right)=\left[\begin{array}{cccc}
-1 & 0 & -1 & 0 \\
0 & -1 & 0 & -1 \\
1 & 0 & 0 & 0 \\
0 & 1 & 0 & 0
\end{array}\right]
$$

have at most two \pm 1 in each row and the other elements 0 . 
Let $t=4$, set $s=1$ then the polynomial $x^{4}+3 x^{2}+1$ has roots $\omega i, \bar{\omega} i,-\omega i$, and $-\bar{\omega} i$. If, in the field $\mathbb{Z}[\omega i]$ we consider the basis $\{1, \omega, i, \omega i\}$, then we have

$$
\rho(\omega i)=\rho(\omega) \rho(i)=\left[\begin{array}{cccc}
0 & 0 & 0 & 1 \\
0 & 0 & 1 & 1 \\
0 & -1 & 0 & 0 \\
-1 & -1 & 0 & 0
\end{array}\right], \quad \rho(i)=\left[\begin{array}{cccc}
0 & 0 & 1 & 0 \\
0 & 0 & 0 & 1 \\
-1 & 0 & 0 & 0 \\
0 & -1 & 0 & 0
\end{array}\right]
$$

From $\rho(\omega i)^{4 m+\epsilon}=\rho(\omega)^{4 m+\epsilon} \rho(i)^{\epsilon}$, with $\epsilon=0,1,2,3$, it follows that $\rho(\omega i)$ is an FM since $\rho(\omega)$ is a block diagonal FM and $\rho(i)$ have at most one \pm 1 in each row and the other elements 0 .

REDUCIBLE APERIODIC FM. Referring to the list (4) of characteristic polynomials for reducible aperiodic FM, we give an example for each case with $s=1$.

(1) $x^{4}-L_{2} x^{2}+1=\left(x^{2}-\omega^{2}\right)\left(x^{2}-\bar{\omega}^{2}\right)=\left(x^{2}-L_{1} x-1\right)\left(x^{2}+L_{1} x-1\right)$, an example is provided by the FM in [3].

(2) $\left(x^{2} \pm L_{1} x+-1\right)^{2}$, an example in the case of sign - is Dazheng matrix $\rho(\omega)$, and for the sign + is $-\rho(\omega)$.

(3) $\left(x^{2} \pm L_{1} x+-1\right)\left(x^{2} \pm 1\right)$,

$$
\left[\begin{array}{llll}
1 & 0 & 0 & 0 \\
0 & 1 & 0 & 0 \\
0 & 0 & 1 & 0 \\
1 & 0 & 0 & 1
\end{array}\right]\left[\begin{array}{cccc}
0 & 1 & 0 & 0 \\
1 & 1 & 0 & 0 \\
0 & 0 & 0 & \pm 1 \\
0 & 0 & 1 & 0
\end{array}\right]\left[\begin{array}{cccc}
1 & 0 & 0 & 0 \\
0 & 1 & 0 & 0 \\
0 & 0 & 1 & 0 \\
-1 & 0 & 0 & 1
\end{array}\right]=\left[\begin{array}{cccc}
0 & 1 & 0 & 0 \\
1 & 1 & 0 & 0 \\
\mp 1 & 0 & 0 & \pm 1 \\
0 & 1 & 1 & 0
\end{array}\right]
$$

(4) $\left(x^{2} \pm L_{1} x+-1\right)\left(x^{2} \pm x+1\right)$,

$$
\left[\begin{array}{llll}
1 & 0 & 0 & 0 \\
0 & 1 & 0 & 0 \\
0 & 0 & 1 & 0 \\
1 & 0 & 0 & 1
\end{array}\right]\left[\begin{array}{cccc}
0 & 1 & 0 & 0 \\
1 & 1 & 0 & 0 \\
0 & 0 & \pm 1 & 1 \\
0 & 0 & -1 & 0
\end{array}\right]\left[\begin{array}{cccc}
1 & 0 & 0 & 0 \\
0 & 1 & 0 & 0 \\
0 & 0 & 1 & 0 \\
-1 & 0 & 0 & 1
\end{array}\right]=\left[\begin{array}{cccc}
0 & 1 & 0 & 0 \\
1 & 1 & 0 & 0 \\
-1 & 0 & \pm 1 & 1 \\
0 & 1 & -1 & 0
\end{array}\right]
$$

4. Conclusions. In this paper, we have shown that the construction of Fibonacci matrices stems from a matrix representation of algebraic number fields containing the golden section ring $\mathbb{Z}[\omega]$. In particular, a construction of $2 n \times 2 n$ FM for any $n$ has been presented, and irreducible FMs of degree 6 and 8 have been shown.

In [9], Washington observed that the set of Fibonacci matrices considered by Dazheng is a representation of the full group of units in the cyclotomic ring $\mathbb{Z}\left[\zeta_{5}\right]$. The matrices of degree 8 obtained here form an analogous subgroup of the units in $\mathbb{Z}\left[\zeta_{15}\right]$; nevertheless they cannot generate the full group of units. An interesting problem that appears to be open is to find all cyclotomic fields whose group of units contains a subgroup in a matrix representation formed by FMs.

Lastly, the classification problem for Fibonacci matrices has been considered, and the classification of the $4 \times 4$ matrices outlined. 


\section{Appendix}

Integral representations of algebraic integer number rings. Let $2 \mathfrak{W}(r, \mathbb{R})$ denote the ring of $r \times r$ matrices with entries from a ring $\mathbb{R}$. An integral representation of the algebraic ring of integers $\mathbb{Z}[\eta]$ is an homomorphism $\Phi: \mathbb{Z}[\eta] \rightarrow \mathfrak{W}(r, \mathbb{Z})$, that is,

$$
\begin{gathered}
\Phi(a \alpha+b \beta)=a \Phi(\alpha)+b \Phi(\beta), \quad \forall a, b \in \mathbb{Z}, \\
\Phi(\alpha \beta)=\Phi(\alpha) \Phi(\beta) .
\end{gathered}
$$

A representation $\Phi$ is said to be faithful if the kernel $\operatorname{Ker}(\Phi)=\{0\}$. The natural representation $\rho$ of $\mathbb{Z}[\eta]$ is a faithful representation defined by

$$
\rho(\eta)=A=\left[\begin{array}{ccccc}
0 & 1 & 0 & \cdots & 0 \\
& \vdots & & \ddots & \\
0 & 0 & 0 & \cdots & 1 \\
e_{r} & e_{r-1} & e_{r-2} & \cdots & e_{1}
\end{array}\right],
$$

where $A$ is the companion matrix of $g(x)=x^{r}-e_{1} x^{r-1}-e_{2} x^{r-2}-\cdots-e_{r-1} x-e_{r}$, the irreducible polynomial with root $\eta$. The image $\operatorname{Im}(\rho)$ of $\mathbb{Z}[\eta]$ is the subring of $2 \mathfrak{W}(r, \mathbb{Z})$ consisting of the matrices of the form $\rho(\alpha)=a_{0} I_{t}+\cdots+a_{r-1} A^{r-1}$. Two integral representations $\rho$ and $v$ of $\mathbb{Z}[\eta]$ are called equivalent if there is a matrix $U$ with rational entries, such that $\rho(x)=U v(x) U^{-1}$ for every $x \in \mathbb{Z}[\eta]$. An integral representation $\rho$ of $\mathbb{Z}[\eta]$ is said to be $\mathbb{Q}$-irreducible if no matrix $Q$ with entries in $\mathbb{Q}$ exists such that $Q \rho(\eta) Q^{-1}$ is a block diagonal matrix with integer entries. A $\mathbb{Q}$ irreducible representation $\rho$ of $\mathbb{Z}[\eta]$ is said to be $\mathbb{F}$-reducible over some extension field $\mathbb{F}$ if there is a matrix $Q$ with entries in $\mathbb{F}$ such that $Q \rho(x) Q^{-1}$ is a block diagonal matrix over $\mathbb{F}$ for all $x \in \mathbb{Z}[\eta]$, otherwise it is said to be absolutely irreducible.

Proposition A.1. The natural representation $\rho$ of $\mathbb{Z}[\eta]$ is $\mathbb{Q}$-irreducible and is completely reducible over the splitting field $\mathbb{E}$ of $g(x)$. If $\mathbb{Z}[\theta]$ is a proper subring of $\mathbb{Z}[\eta]$ of degree $t / r$, then

- $g(x)=\prod_{i=1}^{t} g_{i}(x)$ splits over $\mathbb{Z}[\theta]$ into the product of $t$ irreducible polynomials of the same degree $r / t$

- any natural representation $\rho$ of $\mathbb{Z}[\eta]$ is equivalent to a representation $v$ in which the matrices $v(x)$, with $x \in \mathbb{Z}[\theta]$, are an outer tensor product of the $t \times t$ matrices of a natural representation $\psi$ of $\mathbb{Z}[\theta]$ by the $r / t \times r / t$ identity matrix;

- the characteristic polynomial of a matrix $\rho(x)$ with $x \in \mathbb{Z}[\theta]$ is a power of exponent $r / t$ of the characteristic polynomial of $\psi(x)$.

ACKNowledgement. This paper was presented at the "Ninth International Conference on Fibonacci Numbers and their Applications," Luxembourg, July 17-22, 2000.

\section{REFERENCES}

[1] L. Dazheng, Fibonacci matrices, Fibonacci Quart. 37 (1999), no. 1, 14-20. MR 99m:11011. Zbl 940.11003.

[2] F. W. Dodd, Number Theory in the Quadratic Field with Golden Section Unit, Examples of Mathematical Structures, vol. 3, Polygonal, New Jersey, 1983. MR 85a:11001. Zbl 529.12002. 
[3] P. Filipponi, A family of 4-by-4 Fibonacci matrices, Fibonacci Quart. 35 (1997), no. 4, 300308. MR 98j:11005. Zbl 904.15008.

[4] C. C. MacDuffee, The Theory of Matrices, Chelsea, New York, 1946.

[5] W. Rudin, Principles of Mathematical Analysis, 2nd ed., McGraw-Hill, New York, 1964. MR 29\#3587. Zbl 148.02903.

[6] P. Samuel, Algebraic Theory of Numbers, Houghton Mifflin, Massachusetts, 1970. MR 42 \#177. Zbl 215.36001.

[7] W. Sierpiński, Elementary Theory of Numbers, 2nd ed., North-Holland Mathematical Library, vol. 31, North-Holland, PWN-Polish Scientific Publishers, Amsterdam, Warsaw, 1988. MR 89f:11003. Zbl 638.10001.

[8] L. C. Washington, Introduction to Cyclotomic Fields, 2nd ed., Graduate Texts in Mathematics, vol. 83, Springer-Verlag, New York, 1997. MR 97h:11130. Zbl 0966.11047.

[9] __ Some remarks on Fibonacci matrices, Fibonacci Quart. 37 (1999), no. 4, 333-341. MR 2000j:11040. Zbl 940.11004.

Michele Elia: Dipartimento di Elettronica, Politecnico di Torino, Corso DuCA DeGli ABRUZZI 24, I-10129 TORINO, ITALY

E-mail address: elia@polito.it 


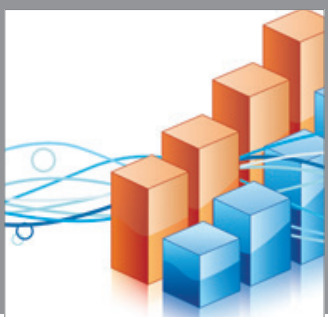

Advances in

Operations Research

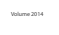

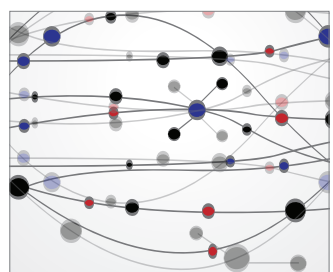

\section{The Scientific} World Journal
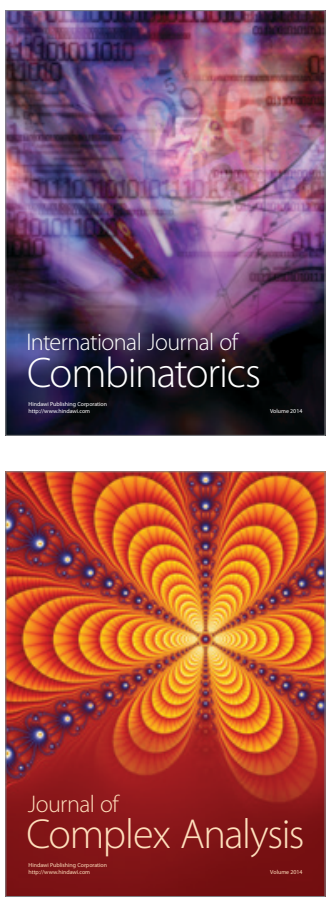

International Journal of

Mathematics and

Mathematical

Sciences
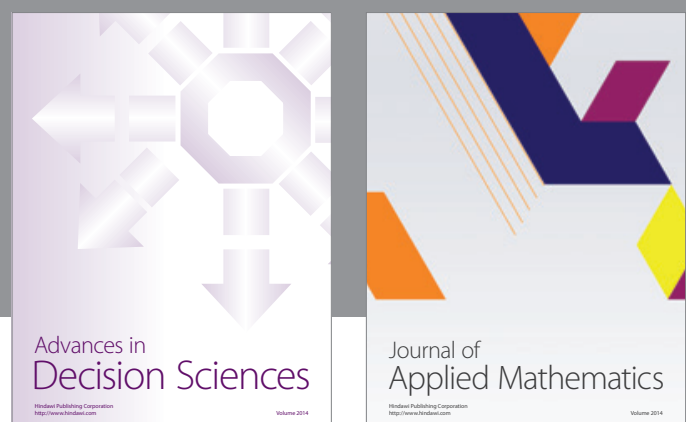

Journal of

Applied Mathematics
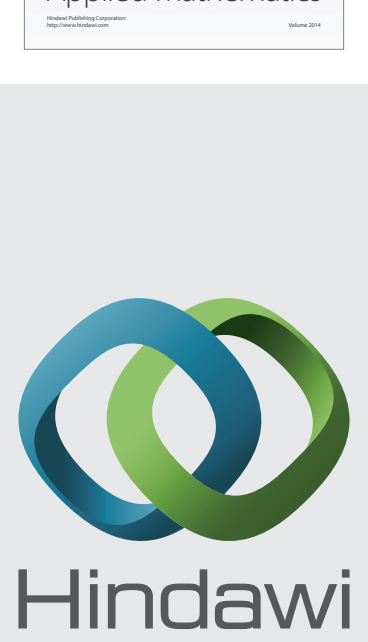

Submit your manuscripts at http://www.hindawi.com
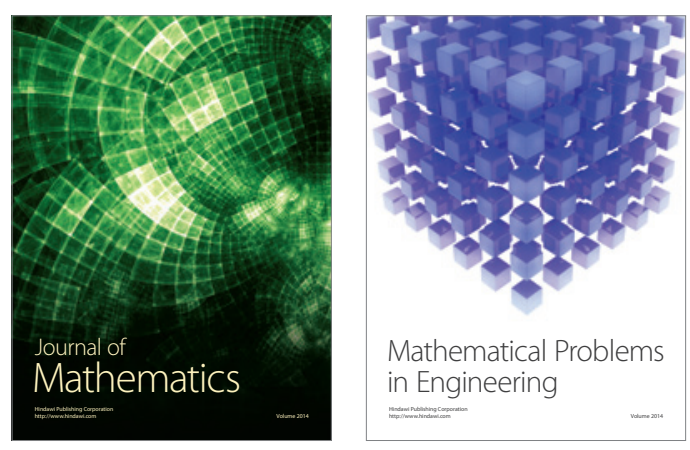

Mathematical Problems in Engineering
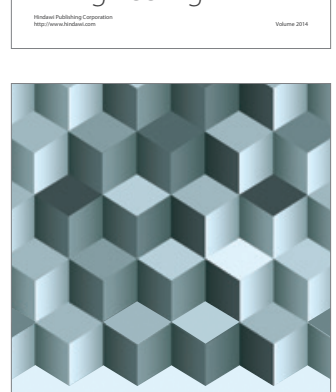

Journal of

Function Spaces
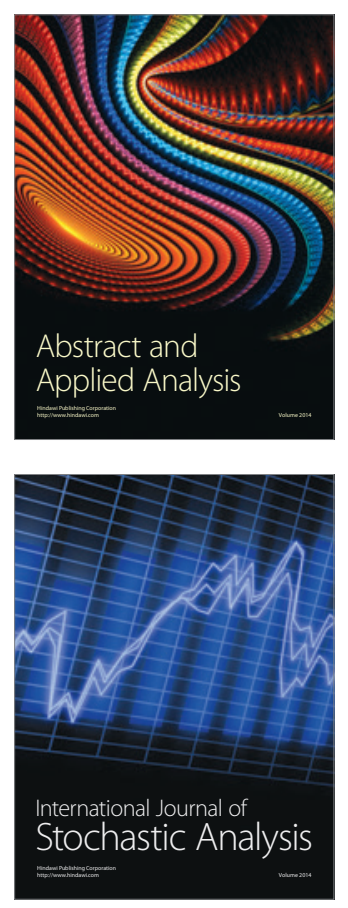

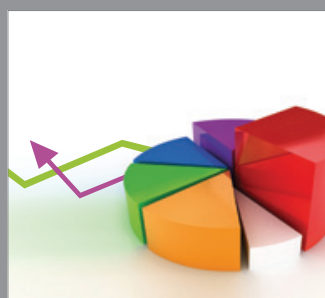

ournal of

Probability and Statistics

Promensencen
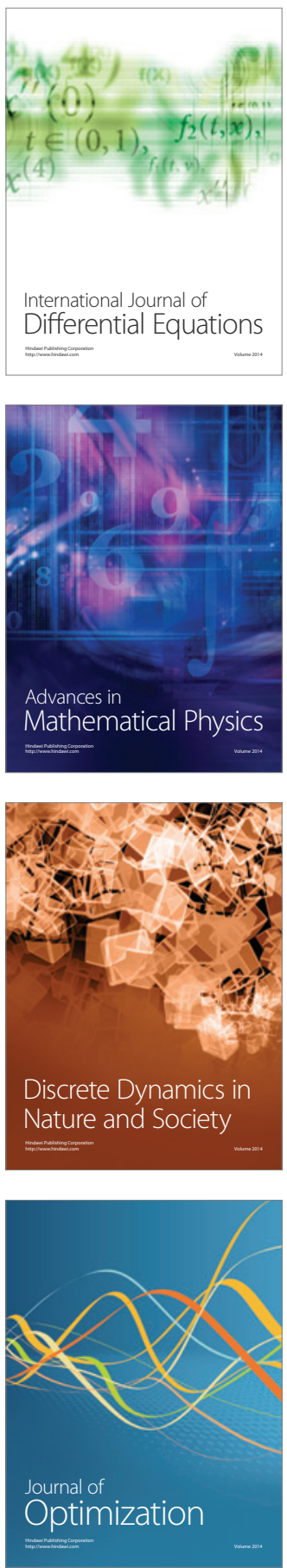